Platform pricing in matching markets

by

Maarten Goos, Patrick Van Cayseele \& Bert Willekens

Monetary and information Economics

Center for Economic Studies

Discussions Paper Series (DPS) 11.32

http://www.econ.kuleuven.be/ces/discussionpapers/default.htm 


\title{
Platform Pricing in Matching Markets
}

\author{
Maarten Goos, Patrick Van Cayseele and Bert Willekens* \\ December 2, 2011
}

\begin{abstract}
This paper develops a simple model of monopoly platform pricing accounting for two pertinent features of matching markets. 1) The trading process is characterized by search and matching frictions implying limits to positive cross-side network effects and the presence of own-side congestion. 2) Matched agents bargain over the division of the match surplus depending on the qualitative characteristics of both parties. We find that, compared to the frictionless benchmark typically analyzed in the classic platform pricing literature, the harms of monopoly market power are mitigated by frictions. However, when the platform is allowed to make investments in the reduction of frictions, a private platform is likely to under-invest compared to a Pigouvian platform. In addition, accounting for user quality differentiation further reduces classic harms of monopoly market power when user quality types are complements in creation of the match surplus. In this case it is socially desirable to attract smaller groups of users with higher average quality to maximize the aggregate match surplus, resulting in a downward price distortion. This result is reversed when quality types are substitutes and the distortion disappears when they are strategically independent.
\end{abstract}

\section{Introduction}

When a worker enters a matchmaking platform, the probability of finding a job is greater when there are many posted vacancies on the platform. However, for a given amount of vacancies, the probability is smaller if more other workers are searching for a job on the platform because only a single applicant can fill each vacancy and therefore workers compete for jobs among each other. The same intuition holds for firms who prefer more workers searching and less other firms posting vacancies. In other words, matching markets are characterized by positive cross-side network effects and negative own-side network effects. Furthermore, the relative presence of both types of

\footnotetext{
*Goos: Catholic University of Leuven (K.U. Leuven) and University College London, maarten.goos@econ.kuleuven.be. Van Cayseele: K.U. Leuven and University of Amsterdam, patrick.vancayseele@econ.kuleuven.be. Willekens: K.U. Leuven, bert.willekens@econ.kuleuven.be. Acknowledgements: We gratefully acknowledge Carlos Cañón, Renato Gomes, Joep Konings, Andras Niedermayer, Jo Van Biesebroeck and numerous seminar and conference participants for useful comments and discussions. Goos and Van Cayseele thank the K.U. Leuven Program Financing/Center of Excellence and Willekens the Research Foundation Flanders for financial support.
} 
network effects crucially depends on the efficiency of the search and matching technology offered by the platform to mitigate the trade imperfections inherent to the matching process. If the technology is more efficient in reducing frictions, the own-side congestion effect is typically smaller and the positive cross-side effect is larger for both sides of the market. A similar intuition applies for platforms that operate in other matching markets like the housing market or the dating market.

The extensive literature on two-sided markets is, however, not directly applicable to platforms operating in matching markets because it investigates the pricing behavior of platforms in markets where trade is nonexclusive (all participants of one side of the market can trade with all participants of the other side and visa-versa). Classic examples (e.g. in Caillaud and Jullien (2003), Rochet and Tirole (2003, 2006) and Armstrong (2006)) are payment cards, video game consoles, newspapers, shopping malls, etc. The main focus is to evaluate the impact of (positive or negative) crossside network externalities on optimal platform pricing behavior under different specifications of user preferences and competitive environments. These models do not explicitly characterize trade imperfections and ignore own-side congestion effects that result from frictions inherent to the trading process ${ }^{1}$.

A first important contribution of this paper is to incorporate search and matching frictions in a simple model of monopoly platform pricing. To do so, we introduce a matching function, which denotes the total number transactions that occur through the platform as a function of the number of participants on both sides of the market and a limited set of parameters that reflect, for example, the efficiency of the matching technology under which the platform operates. The virtue of introducing a general matching function is that it allows us to characterize frictions inherent to matching markets in a general and tractable way without making explicit the specific market imperfections behind them ${ }^{2}$. It is shown that the relative presence of positive cross-side and negative own-side network externalities endogenously follows from the second-order properties of the matching function, reflecting the amount of trading frictions that characterize the trading process and the way the platform is able to mitigate the inefficiencies that go hand in hand with these frictions ${ }^{3}$.

\footnotetext{
${ }^{1} \mathrm{~A}$ recent development in the platform pricing literature is to reconcile the seminal contributions of, for example, Caillaud and Jullien (2003), Rochet and Tirole (2003, 2006) and Armstrong (2006) into generalized models that allow for arbitrary specifications of user preferences (e.g. Weyl (2010) and Veiga and Weyl (2011)) and platform competition (e.g. Weyl and White (2011)). Implicitly these general models allow for many market imperfections, but it is impossible to make inference about the impact of these imperfections on the model outcomes without imposing additional assumptions or explicit functional forms.

${ }^{2}$ We borrow this concept from the search and matching literature mainly applied to labor markets. See Pissarides (2000) and Rogerson, Shimer and Wright (2005) for an overview of how the matching function is put to work in labor market theory. See Petrongolo and Pissarides (2001) for a survey on microfoundations and empirical estimation of the matching function.

${ }^{3}$ Other contributions in the platform pricing literature that have focussed on own-side network externalities are, for example, Belleflamme and Toulemonde (2009), Viecens (2009) and Anderson and de Palma (2009). However, none of these contributions directly link the relative presence of own-side and cross-side externalities to the amount of trading frictions present in the market. A notable exception is Cheng and Huang (2009) who use a specific matching function to model the matching process. Their model can be considered a special case of our more
} 
In this environment we analyze and compare the optimal pricing and investment decisions of a profit and social value maximizing platform. As in Weyl (2010) we find that in the optimal pricing decision the private platform only accounts for the private value of the network externalities, while the Pigouvian platform accounts for the social value, resulting in a Spence distortion. In contrast to Weyl (2010), however, in our framework the Spence distortion has two specific components. An upward cross-side network size distortion and a downward own-side network size distortion. Therefore, the harms of monopoly market power are mitigated in the presence of frictions compared to the frictionless benchmark typically analyzed in the classic platform pricing models. When allowing the platform to invest in the reduction of trading frictions, we also find that for conventional weakly convex investment-costs and decreasing marginal gains in matching efficiency, the private platform under-invests in matching technologies compared to the Pigouvian platform.

A second contribution of the paper is to extend the model such that transaction valuations are no longer considered exogenous. Instead, users are allowed to care about the characteristics of potential trading partners and bargain over the division of the match surplus. This is another distinctive feature of matching markets which has received little attention in the platform pricing literature. Therefore, we introduce a general match value function which denotes the total match surplus depending on the quality type of two matched agents ${ }^{4}$. Accounting for user quality differentiation results in an additional quality externality on top of the two network size externalities discussed above. The intuition is that because a marginal user attracted on a particular side is of inferior quality compared to the inframarginal users on that side the average quality of the pool degrades. This results in a negative externality because the expected match surplus of users on the other side decreases.

We compare again how a private and social value maximizing platform account for this in their optimal pricing decisions. This results in an additional component in the Spence distortion, referred to as the quality distortion. A direct link is established between the sign and magnitude of the quality distortion and the cross-partial of quality types in the match value function. As is well-

general approach.

${ }^{4}$ Crucial for our results is that we impose minimal restrictions on the match value function in the sense that user quality types are allowed to be both complements, substitutes or strategically independent. This in contrast to the other contributions in the platform pricing literature that allow the transaction surplus to depend on the quality characteristics of both trading partners (e.g. Damiano and Li $(2007,2008)$ and Trégouët (2010)) who restrict the match value function to be supermodular and multiplicatively separable. Supermodularity implies the quality types of matched users are always complements. As discussed in greater detail in section 4, these assumptions can be overly restrictive and are not necessary in our setup.

Compared to our general specification of the match value function, Veiga and Weyl (2011) impose even less restrictions on the payoff functions of participating users. Users are allowed to value and contribute to an arbitrary amount of platform characteristics (of which quality can be one). However, they do not discuss the implications of a generic match value function in a multi-sided setting and do not establish the direct link between the strategic dependence of user quality types and the direction of market distortions that result from vertical quality differentiation in a context of matching. 
known from the matching literature, the cross-partial reveals whether and to which extent quality types are complement or substitutes and serves as a measure of the social value of sorting users by type in matching markets ${ }^{5}$. For complements (substitutes) the quality distortion is downward (upward) and it disappears for strategically independent quality types, which is for example the case if user utility is nontransferable ${ }^{6}$.

A third important contribution is that our approach suggests that techniques and methods used in the rich empirical search and matching literature can be used to identify parameters important for platform pricing and investment decisions and the distortions that potentially result from market power. For example, under some simplifying assumptions, by simply tracking user entry and exit over time, the amount of trading frictions present at the platform can be estimated ${ }^{7}$. In addition, in markets with exclusive trade (e.g. labor market, housing market) important determinants of user payoffs are ex post observed (e.g. wages, housing prices) and can be used to make inference about user preferences and properties of the matching process. This as opposed to the canonical examples of two-sided markets (e.g. newspapers, payment cards) where user valuations for the platform service are typically unobserved.

The remainder of this article is organized as follows. Section 2 presents the basic model assumptions and sections 3 and 4 derive the main results for exogenous and endogenous match valuations, respectively. Section 5 discusses the critical assumptions and links our results to other closely related work. Section 6 concludes. All results in the body of the paper are derived for unidimensional user heterogeneity. The implications on the results for richer specifications of user preferences as in Weyl (2010) and Veiga and Weyl (2011) are described in the appendix.

\section{Basic model assumptions}

\subsection{Setup, timing and user preferences}

Consider a monopoly platform through which participants of two types of risk-neutral user groups $I=A, B$ can potentially interact. The mass of potential users on each side is normalized to unity. Users of the two sides can only interact through the platform and the payoff of not participating is normalized to zero. The fraction of potential users that patronize the platform on side $I$ is denoted by $N^{I}$. The platform charges a uniform participation fee $P^{I}$ to users on side $I=A, B$, i.e. the platform can third-degree price discriminate between, but not within groups.

We analyze a three-stage static game. In the first stage the platform announces prices and

\footnotetext{
${ }^{5}$ See, for example, Becker (1973), Shimer and Smith (2000) and Eeckhout and Kircher (2010, 2011) for a discussion.

${ }^{6}$ Cañón (2011) and Gomes and Pavan (2011) assume nontransferable utility in the sense that transaction valuations only depend on the qualitative characteristics of the trading partner. Therefore in their welfare implications they do not find similar quality distortions as in the present paper.

${ }^{7}$ See Petrongolo and Pissarides (2001) for a review of estimation of properties of the matching function in aggregate labor markets.
} 
the matching function $M$ under which it operates. In the second stage potential users on both sides simultaneously decide on participation. Users that decide to patronize the platform pay the participation fee. In the third stage, $M$ transactions occur through the platform.

Formally, individual expected utility (at stage 2) of users on side $I=A, B$ of patronizing the platform is given by

$$
u^{I}=v^{I} \frac{M}{N^{I}}-P^{I} .
$$

Users are heterogeneous in their valuation of a match with a user on the other side, denoted by $v^{I}$, distributed over a twice continuously differentiable distribution function $F^{I}($.$) and density$ function $f^{I}($.) with nonnegative support. Individual match valuations are exogenous and private information, but the distributions are common knowledge. Note that $u^{I}=0 \Leftrightarrow v^{I}=\frac{P^{I} N^{I}}{M} \equiv \widetilde{v^{I}}$ where $\widetilde{v^{I}}$ is defined as the match valuation of the marginal user that participates on side $I$.

In this simple specification of user utility we make the following important assumptions. To start, user heterogeneity is unidimensional in match valuations and there are no fixed benefits of patronizing the platform. We argue that for a matchmaking platform this assumption is not overly restrictive because typically there is little to gain by users besides finding a valuable match and hence little heterogeneity is expected in fixed user benefits to enter the platform ${ }^{8}$. In addition, we restrict match valuations to be nonnegative because in matching markets transactions can typically not be enforced. Users with a negative valuation refuse all matches and act as if they have a match valuation of zero. They only participate when being subsidized, i.e. if $P^{I} \leq 0^{9}$.

A more fundamental assumption is that matching is ex ante random. Users expect the total amount of transactions to be divided with equal probability over the participants on each side. In other words, when deciding to participate users know their willingness to pay for a transaction and know the size of the platform in terms of the aggregate number of transactions. However, they only discover how trading patterns are exactly established once they enter the platform and for this they first have to incur the participation fee. The trading process is left unspecified and assumed to be realized in a decentralized way for a given search and matching technology offered by the platform. Note that ex post it is perfectly feasible that users with higher match valuations are involved in more transactions or have a higher probability of finding a match relative to those users with lower valuations or visa-versa, but it is assumed that potential users do not have this information when they decide on participation. Consistent with the intuition of decentralized trade, it is assumed that payments conditional on matching are infeasible. This because transactions are simply not observed by the platform or because they cannot be enforced ${ }^{10}$.

\footnotetext{
${ }^{8}$ This in contrast to other classic examples of platforms like newspapers or shopping malls. For example, newspaper readers might significantly differ in their valuation for the content of articles independent of the number of advertisers, or consumers in shopping malls might heterogeneously value a nearby parking lot.

${ }^{9}$ These simplifying assumptions on user heterogeneities are made for a straightforward interpretation of our results, but can easily be relaxed as formally described in the appendix.

${ }^{10}$ Many platform intermediated markets match this description well. For example, platforms in the online labor
} 


\subsection{The matching function, frictions and externalities}

If $N^{A}, N^{B}$ users participate on sides $A, B$, respectively, the total number of transactions realized through the platform is given by the matching function $M=M\left(N^{A}, N^{B}\right)$. We impose the following properties on the matching function: $M\left(N^{A}, N^{B}\right)$ for both arguments is (i) twice continuously differentiable, (ii) weakly increasing and (iii) weakly concave. In addition, (iv) $M\left(N^{A}, 0\right)=$ $M\left(0, N^{B}\right)=0$ and $(\mathrm{v}) M \leq N^{A} N^{B}$.

Property (i) is technical to ensure our model is analytically tractable. Properties (ii), (iii), (iv) and (v) ensure that an additional user on any side can never reduce the number of transactions that occur through the platform, that users of the same side compete for transactions, that at least one user needs to be present at the platform on each side for a transaction to be feasible and that a user of one side can at most interact once with each user on the other side, respectively ${ }^{11}$.

Instead of property (v), in the matching literature it is typically assumed that $M \leq \min \left[N^{A}, N^{B}\right]$ such that trade is exclusive consistent with one-to-one matching. In this case, the ratio $M / N^{I}$ is the probability of finding a match through the platform for users on side $I$ when they make their participation decision. However, we also allow for $M>\min \left[N^{A}, N^{B}\right]$ in which case trade is nonexclusive consistent with a many-to-many matching setting. The ratio $M / N^{I}$ then denotes the expected number of transactions per participant on side $I$ at stage 2 . In what follows we refer to the ratio $M / N^{I}$ as the expected number of transactions, but one should keep in mind that it can be less than one in which case it actually reflects the probability of finding a single match.

In the classic platform pricing models (e.g. Rochet and Tirole (2003, 2006) and Armstrong (2006)) it is assumed that $M=N^{A} N^{B}$, which is the limit case of our matching function referred to as the frictionless benchmark. In this case, the expected number of transactions $M / N^{I}$ of users

(e.g. Monster.com), housing (e.g. ForSaleByOwner.com) or dating market (e.g. Match.com) typically charge participants on one or both sides a fixed participation fee and then offer a certain search technology that can be used by participants to search for one another in a decentralized way. Furthermore, the intuition that users only have imperfect information about exact trading patterns that will occur through the platform when they decide to enter, not only applies to these specific online matching markets, but also applies for most canonical example markets of the platform pricing literature such as newspapers, payment cards, shopping malls, etc. For example, when an advertiser places an ad in a newspaper it has a good idea about the number of readers and the total space reserved for ads in the newspaper. However, it does not exactly know how many "eyeballs" the ad will capture because this also depends on the content of articles, the prominence of competing ads and the way these two are arranged in the final edit of the newspaper, which is only revealed to the advertiser ex post when the advertisement fee is already sunk.

${ }^{11}$ Assumptions (ii)-(v) facilitate the interpretation of our results, but can be relaxed if necessary. For example, relaxing assumption (iii) and allowing the matching function to be convex would imply that the expected number of transactions $M / N^{I}$ can depend positively on own-side participation. In this case $v^{I}$ can no longer be interpreted as the valuation of a transaction with a user of the other side, but would be the valuation of transactions with crossside and own-side users at the same time. This would make the interpretation of our results less straightforeward because the strict separation of the two sides no longer holds. The presence of positive own-side network effects might be realistic in some platform markets (e.g. video game platform on which gamers can play against each other), but then an additively seperable specification of utility in participation rates is likely to be more realistic. Instead the focus of this paper goes to markets where users of the same side compete for transactions and the frictions that this brings about. 
on side $I$ is $N^{J}$. In other words, each user of one side makes exactly one transaction with each user on the other side implying the absence of any trade imperfections.

The virtue of introducing a more general matching function is that it allows us to account for trading frictions in a tractable way without having to make explicit assumptions on the specific market imperfections behind them. Instead, if for whatever reason the matching function diverges more from the frictionless benchmark ( $M$ smaller for any allocation), the market is said to be characterized by greater frictions.

What is important, is that we allow the matching function to be nonlinear - and more specifically concave. Together with the assumption that $M$ is weakly increasing in both arguments, concavity implies that the expected number of transactions $M / N^{I}$ is increasing in cross-side participation $N^{J}$, but with diminishing returns, and is decreasing in own-side participation $N^{I}$. In other words, on each side there are limits to utility derived from cross-side participation and there is own-side congestion because of competition between own-side users for transactions.

A useful measure to quantify these effects is the matching elasticity, defined for side $I=A, B$ as

$$
\varphi^{I} \equiv \frac{\partial M}{\partial N^{I}} \frac{N^{I}}{M}
$$

which, for a given allocation, reflects the degree of concavity of the matching function with respect to participation on side $I$ on scale of zero to one, i.e. $0 \leq \varphi^{I} \leq 1$. Furthermore, as already suggested by Petrongolo and Pissarides (2001), there is a one-to-one relationship between the matching elasticities and the relative presence of own-side and cross-side externalities in the market. We now formalize this argument for our static framework.

The participation decision of a marginal $I$-side user has an impact of $\frac{\partial}{\partial N^{I}}\left(\frac{M}{N^{I}}\right)=\frac{1}{N^{I}}\left(\frac{\partial M}{\partial N^{I}}-\frac{M}{N^{I}}\right)$ on the expected number of transactions of each other $I$-side user. Adding up this effect over all I-side users yields the total amount of negative own-side externalities or congestion induced by the participation decision of a marginal $I$-side user:

$$
\frac{\partial M}{\partial N^{I}}-\frac{M}{N^{I}} \text { or }\left(\varphi^{I}-1\right) \frac{M}{N^{I}}
$$

In addition, a marginal user on side $I$ has an impact of $\frac{\partial}{\partial N^{I}}\left(\frac{M}{N^{J}}\right)=\frac{1}{N^{J}} \frac{\partial M}{\partial N^{I}}$ on the expected number of transactions of each $J$-side user. Adding up over all $J$-side users yields the total amount of positive cross-side externalities induced by a marginal $I$-side user:

$$
\frac{\partial M}{\partial N^{I}} \text { or } \varphi^{I} \frac{M}{N^{I}}
$$

Expressions (2) and (3) illustrate that attracting a marginal user on either side $I=A, B$ has two effects. First, it results in additional transactions measured by $\frac{\partial M}{\partial N^{I}}$, which is positive for both sides. Second, it also has a negative effect because it reduces the expected number of transactions 
of all other users on the same side, $\frac{M}{N^{I}}$, because of more fierce competition for transactions. For a concave matching function the positive marginal effect is always smaller than the negative average effect, implying the presence of negative own-side externalities.

In addition, expressions (2) and (3) illustrate the one-to-one relationship between the matching elasticities for each side, and the relative presence of own-side and cross-side network externalities at that side. A smaller elasticity implies more own-side congestion and less positive cross-side effects. Note that for the frictionless benchmark $M=N^{A} N^{B}$, the matching elasticities satisfy $\varphi^{A}=\varphi^{B}=1$. So, there is no congestion and only positive cross-side network externalities measured by $N^{J}$.

To be clear, a greater presence of trading frictions in the market might be reflected in a smaller matching elasticity on one or both sides, in which case they imply greater own-side congestion and less positive cross-side externalities. However, frictions might also reduce the aggregate number of transactions, while leaving the matching elasticities unaltered. To see this, consider the popular Cobb-Douglas specification of the matching function often used in the empirical matching literature of the form $M=\gamma\left(N^{A}\right)^{\varphi^{A}}\left(N^{B}\right)^{\varphi^{B}}$. In this case, the amount of frictions present in the market can be estimated through three parameters, a linear component $\gamma$ and two nonlinear components $\varphi^{A}$ and $\varphi^{B}$. For the Cobb-Douglas specification matching elasticities $\varphi^{A}$ and $\varphi^{B}$ are constant, however, this does not have to be the case for our more general specification of the matching function. For a review on estimating properties of the matching function for aggregate labor markets and of other specific functional forms of the matching function used in the matching literature see Petrongolo and Pissarides (2001).

\section{Solving the model}

\subsection{Demand, multiplicity and insulating tariffs}

Users on side $I$ participate if $u^{I} \geq 0$ for a given participation fee, matching technology and crossside participation rate. Quasi-demand is given by $N^{I}=1-F^{I}\left(\widetilde{v^{I}}\right)$ and given our assumptions of smoothness of the matching and distribution functions the inverse demand functions for sides $I=A, B$ are well-defined:

$$
P^{I}\left(N^{I}, N^{J}\right)=F^{I^{-1}}\left(1-N^{I}\right) \frac{M\left(N^{I}, N^{J}\right)}{N^{I}}
$$

It is well-known from the two-sided markets literature that if the platform chooses optimal prices at the first stage and users of the two sides simultaneously decide on participation in the second stage, there is an inherent problem of equilibrium multiplicity due to user coordination failures $^{12}$. However, as argued by Weyl (2010), the platform can eliminate the coordination problem

\footnotetext{
${ }^{12}$ See, for example, Caillaud and Jullien (2003), Weyl (2010), Weyl and White (2011) and Veiga and Weyl (2011) for a discussion.
} 
by directly choosing a desired allocation instead of indirectly through prices. Because the inverse demand function (4) uniquely pins down the $I$-side price for any allocation, the platform can make the participation decision of $I$-side users invariant to the participation decision of $J$-side users by simply adjusting the price on side $I$ to variations in $J$-side participation. In other words, the platform on each side can insulate any desired participation rate by setting the price contingent on cross-side participation. Therefore, Weyl (2010) refers to the unique set of prices that follow from the chosen allocation of the platform as insulating tariffs. Consistently, in what follows we assume the platform maximizes its objective function by choosing an optimal allocation.

\subsection{Private platform pricing}

The profit maximization problem of a monopoly private platform can be summarized as follows ${ }^{13}$,

$$
\max _{N^{A}, N^{B}} \pi=P^{A}\left(N^{A}, N^{B}\right) N^{A}+P^{B}\left(N^{A}, N^{B}\right) N^{B}-C\left(N^{A}, N^{B}\right)
$$

where $P^{I}\left(N^{I}, N^{J}\right)$ is given by (4) and $C\left(N^{A}, N^{B}\right)$ is the platform cost function to attract users on sides $I=A, B$, assumed twice continuously differentiable in both arguments. Denote $C_{N^{I}} \equiv \frac{\partial C}{\partial N^{I}}$ as the marginal cost of attracting an additional $I$-side user and $\mu_{I} \equiv \frac{1-F^{I}\left(\widetilde{v^{I}}\right)}{f^{I}\left(\widetilde{v^{I}}\right)}$ as the inverse hazard rate of demand. Proposition 1 directly follows from the first-order conditions of (5).

Proposition 1 For each side $I=A, B(J \neq I)$, the optimal markup of price over marginal costs of a private monopoly platform is given by

$$
P^{I}-C_{N^{I}}=\mu^{I} \frac{M}{N^{I}}-\underbrace{\widetilde{v^{J}} \varphi^{I} \frac{M}{N^{I}}}_{\begin{array}{c}
\text { Subsidize positive cross-side } \\
\text { network externalities }
\end{array}}+\underbrace{\widetilde{v^{I}\left(1-\varphi^{I}\right) \frac{M}{N^{I}}}}_{\begin{array}{c}
\text { Tax negative own-side } \\
\text { network externalities }
\end{array}},
$$

Proposition 1 demonstrates that on each side $I=A, B$ the optimal price over marginal costs of a monopoly private platform can be subdivided in three components. The first term on the right hand side of expression (6) shows that the inverse hazard rate of demand, which is the classic measure of monopoly market power, in our setting is multiplied by the expected number of transactions. For the frictionless matching function $M=N^{A} N^{B}, M / N^{I}=N^{J}$ and frictions imply that $M / N^{I}<N^{J}$. So, the presence of more frictions imply a greater reduction in classic market power.

\footnotetext{
${ }^{13}$ The last step to ensure a unique solution to the proposed three-stage game is to investigate whether or not there actually is a unique allocation chosen by the platform when optimizing its objective function. In our setup, the conditions under which the profit maximization problem satisfies local concavity with respect to the optimal set $\left\{N^{A}, N^{B}\right\}$ are equivalent to the two-sided contraction conditions proposed by Weyl (2010). Notes are available on request. In what follows we assume that the conditions for local concavity of the platform objective function are satisfied.
} 
The second term illustrates how the platform on each side accounts for the positive cross-side network externalities (measured by $\varphi^{I} \frac{M}{N^{I}}$ as shown in expression (3)) present in the market. The valuation of the marginal $J$-side user of the entry of a marginal $I$-side user enters the optimal platform pricing equation for side $I$ with a negative sign. The platform grants this subsidy to $I$-side users because it is exactly the marginal revenue it can recoup on side $J$ from lowering the price on side $I$ to attract an additional $I$-side user. The subsidization of cross-side externalities is a standard result in the existing platform pricing literature. However, we show that the subsidy is smaller in the presence of frictions (reflected in a smaller $\varphi^{I}, M / N^{I}$ or both) because they imply reduced presence of cross-side externalities.

The third term is particular to our setting and reflects how the platform accounts for negative own-side network externalities (measured by $\left(\varphi^{I}-1\right) \frac{M}{N^{I}}$ as shown in expression (2)). As discussed in section 2.2, a matching elasticity less than one implies the presence of own-side congestion on side $I$ because the gain of attracting an additional $I$-side user cannot compensate the reduction in the expected number of transactions of $I$-side users. The private platform takes this into account in its pricing decision on each side by taxing the negative own-side externality proportional to the match valuation of the marginal users on that side. The platform imposes this tax to recover the loss in marginal revenue induced by the congestion of the marginal user.

Overall, proposition 1 shows that not accounting for trading frictions when needed, and the congestion effects that possibly result from them, can result in severely biased predictions about the optimal private platform pricing decisions.

\subsection{Pigouvian pricing and price distortions}

As first pointed out by Spence (1975), a monopoly that decides both on price (or quantity) and product quality tends to serve the quality preferences of marginal users instead of average users as would be optimal from a social point of view. Weyl (2010) revisits this argument for multi-sided platforms by interpreting the amount of users on side $J$ as a measure of quality of the platform service for users on side $I$. Weyl (2010) finds similar results to Spence (1975) in the sense that a monopoly platform in its optimal pricing decision tends to internalize network externalities with respect to the valuation of marginal users instead of average users. Consistently, in this section we investigate the impact of trading frictions on price distortions by interpreting the expected number of transactions on each side as a measure of quality of the platform service. Price distortions are identified by comparing the pricing decisions of a private platform to those of a social planner.

A Pigouvian platform maximizes total social value

$$
\max _{N^{A}, N^{B}} V=\left(\overline{v^{A}}+\overline{v^{B}}\right) M-C\left(N^{A}, N^{B}\right)
$$


where $\overline{v^{I}}$ measures the average match valuation of participating users on side $I=A, B$,

$$
\overline{v^{I}} \equiv \frac{1}{N^{I}} \int_{\widetilde{v^{I}}}^{+\infty} v^{I} f^{I}\left(v^{I}\right) d v^{I}
$$

and hence $\left(\overline{v^{A}}+\overline{v^{B}}\right) M$ measures the total user surplus that results from the platform service. From the first-order conditions of (7), the markup of price over marginal costs of the Pigouvian platform for each side $I=A, B(J \neq I)$ can easily be derived,

$$
P_{P i g}^{I}-C_{N^{I}}=-\underbrace{\overline{v^{J}} \varphi^{I} \frac{M}{N^{I}}}_{\begin{array}{c}
\text { Subsidize positive cross-side } \\
\text { network externalities }
\end{array}}+\underbrace{\overline{v^{I}}\left(1-\varphi^{I}\right) \frac{M}{N^{I}}}_{\begin{array}{c}
\text { Tax negative own-side } \\
\text { network externalities }
\end{array}} .
$$

From expression (8) it is clear that the Pigouvian platform internalizes network externalities with respect to the average match valuation of all users participating on the platform and not just with respect the match valuation of the marginal user as the private platform does. By adding to and subtracting from the expression of the optimal private markup (6) the terms of the right hand side of expression (8), the price distortions that result from monopoly market power are conveniently illustrated, as summarized in proposition 2.

Proposition 2 For each side $I=A, B(J \neq I)$, the optimal price of the private monopoly platform can be written as,

$$
P^{I}=\underbrace{P_{\text {Pig }}^{I}}_{\begin{array}{c}
\text { Socially } \\
\text { optimal price }
\end{array}}+\underbrace{\mu^{I} \frac{M}{N^{I}}}_{\begin{array}{c}
\text { Classic } \\
\text { distortion }
\end{array}}+\overbrace{\begin{array}{c}
\text { Cross-side } \\
\text { network size distortion }
\end{array}}^{\overbrace{\left(\overline{v^{J}}-\widetilde{v^{J}}\right) \varphi^{I} \frac{M}{N^{I}}}^{\text {Spence distortion }}}-\underbrace{\left(\overline{v^{I}}-\widetilde{v^{I}}\right)\left(1-\varphi^{I}\right) \frac{M}{N^{I}}}_{\begin{array}{c}
\text { Own-side } \\
\text { network size distortion }
\end{array}} .
$$

Proposition 2 shows that the diverging incentives of the private and Pigouvian platform to account for network externalities results on top of the classic distortion of quantities in, what Weyl (2010) refers to as, a Spence distortion. Note that for the frictionless benchmark $M=N^{A} N^{B}$ it is equal to $\left(\overline{v^{J}}-\widetilde{v^{J}}\right) N^{J}$ for the price on side $I$. However, in the presence of congestion effects induced by trading frictions, the Spence distortion has two components to which we refer as the cross-side network size distortion and the own-side network size distortion.

The cross-side network size distortion is upward, implying that the subsidization of the private platform of cross-side network externalities is too small compared to the Pigouvian platform. The subsidy should be proportional to the average match valuation of participating user and as a result the private price is too high compared to what is socially optimal. The upward distortion is smaller in the presence of more frictions. Similarly, the own-side network size distortion is downward because the private platform taxation of negative own-side externalities is too small 
compared to the Pigouvian platform. The own-side network size distortion is more downward if frictions imply more own-side congestion, measured by a smaller matching elasticity $\varphi^{I}$.

Allowing for multiple sources of user heterogeneity in user valuations for the platform service (e.g. in both transaction valuations and fixed benefits of entering the platform) as in Weyl (2010), implies that the average match valuation spreads $\overline{v^{I}}-\widetilde{v^{I}}$ between average and marginal users can also be negative for both sides $I=A, B$ (see appendix). This is more likely to be the case in markets where users are mainly heterogeneous in fixed benefits and where the users with high (low) fixed benefits are also the users with low (high) transaction valuations (e.g. newspaper market where users who highly value content are also the ones who dislike advertisements the most). For the frictionless benchmark this implies that the Spence distortion can be downward and hence can potentially mitigate the classic distortion as suggested by Weyl (2010).

However, as argued above, in one-to-one matching markets users are unlikely to primarily differ in fixed benefits of entering the platform and the match valuation spread $\overline{v^{I}}-\widetilde{v^{I}}$ for both sides is expected to be positive. Nevertheless, proposition 2 demonstrates that in this case the Spence distortion can be downward and this is more likely to be the case in the presence of more own-side congestion.

Overall, proposition 2 shows that a greater presence of trading frictions, which implies $M / N^{I}$ and/or $\varphi^{I}$ are smaller for any allocation, tends to reduce price distortions (and might even result in a downward distortion if the congestion effect is large) compared to the frictionless benchmark. In other words, not accounting for trading frictions when needed unambiguously results in upward biased predictions about the harms of monopoly market power.

\subsection{Platform investment incentives}

So far, the amount of frictions present in the market has been considered exogenous. However, in many platform intermediated markets platforms regularly make investments to improve the efficiency of the matching process of users of the two sides of the market ${ }^{14}$. We define a platform investment to improve efficiency if for any allocation the number of transactions that occur through the platform weakly increases. Or put differently, if the amount of frictions present in the market weakly decrease.

From the Pigouvian platform objective function (7) it is clear that total user surplus $\left(\overline{v^{A}}+\overline{v^{B}}\right) M$ increases in efficiency. However, from the inverse demand equation (4) it is also clear that a more efficient platform can charge a higher price to attract the same amount of users on each side. In addition, investments in efficiency are likely to be costly. Accounting for all these factors it is not

\footnotetext{
${ }^{14}$ For example, on February 1, 2010 on webpronews.com: "Monster talks up its new search technology. Monster Semantic 6 Sense Search working for employers/job seekers". Or on April 26, 2011 on Realtor.com: "REALTOR.com launches new iPad App" ... "now 17 million iPad users have access to an app which makes their next home search easier, faster, and more streamlined than ever before."
} 
trivial that the investment decision of a private platform coincides with the investment decision of the Pigouvian platform.

To investigate this issue, assume there is a generic component $\alpha$ in the matching function that reflects the matching technology under which the platform operates and that positively affects the efficiency of the matching process, i.e. $M_{\alpha} \equiv \frac{\partial M}{\partial \alpha} \geq 0$. In addition, assume investment is costly and the platform cost function becomes $C\left(\alpha, N^{A}, N^{B}\right)$, assumed weakly increasing in $\alpha$, i.e. $C_{\alpha} \equiv \frac{\partial C}{\partial \alpha} \geq 0$, over a nonnegative support.

In addition to the allocation decision the platform is now also allowed to make an investment decision in the first stage. The private platform profit maximization problem becomes,

$$
\max _{\alpha, N^{A}, N^{B}} \pi=P^{A}\left(\alpha, N^{A}, N^{B}\right) N^{A}+P^{B}\left(\alpha, N^{A}, N^{B}\right) N^{B}-C\left(\alpha, N^{A}, N^{B}\right) .
$$

Similarly, the Pigouvian social value maximization problem becomes,

$$
\max _{\alpha, N^{A}, N^{B}} V=\left(\overline{v^{A}}+\overline{v^{B}}\right) M\left(\alpha, N^{A}, N^{B}\right)-C\left(\alpha, N^{A}, N^{B}\right) .
$$

Note that substituting the definition of the match valuation of the marginal user $\widetilde{v^{I}} \equiv \frac{P^{I} N^{I}}{M}$ in the inverse demand equation (4) and rewriting yields $\widetilde{v^{I}}=F^{I^{-1}}\left(1-N^{I}\right)$, which is independent of $\alpha$. By definition $\overline{v^{I}}$ is then also independent of $\alpha$. Therefore, the derivation of the first order conditions for the investment decisions from (9) and (10) is straightforward, as summarized in proposition 3 .

Proposition 3 The optimal investment level $\alpha^{\pi}$ of a private monopoly platform satisfies

$$
\frac{C_{\alpha^{\pi}}}{M_{\alpha^{\pi}}}=\widetilde{v^{A}}+\widetilde{v^{B}}
$$

The optimal investment level $\alpha^{V}$ of a Pigouvian monopoly platform satisfies

$$
\frac{C_{\alpha^{V}}}{M_{\alpha^{V}}}=\overline{v^{A}}+\overline{v^{B}}
$$

Proposition 3 shows that in the optimal investment decision the private platform equates the relative marginal investment-cost over the marginal gain in matching efficiency to the sum of the transaction valuation of the marginal users on the two sides. In contrast, the Pigouvian platform equates the marginal cost over marginal gain to the sum of average match valuations of all participating users on both sides.

So, market distortions that result from platform investment decisions are again related to the match valuation spreads $\overline{v^{I}}-\widetilde{v^{I}}$ which closely hinge on the degree of user heterogeneities on both sides of the market. Under our assumption of unidimensional user heterogeneity, $\overline{v^{I}} \geq \widetilde{v^{I}}$ for both 
sides $I=A, B$, such that the right-hand side of expression (11) is larger or equal to the right-hand side of expression (12). It follows that the private platform over- or under-investment compared to the Pigouvian platform crucially depends on the second-order properties of the investment-cost and matching function.

For example, under the assumption of a convex investment-cost function and a concave matching function, $\frac{C_{\alpha}}{M_{\alpha}}$ is an increasing function and it follows from proposition 3 that in this case $\alpha^{\pi} \leq \alpha^{V}$. So, the private platform under-invests in matching technology and this under-investment is larger if the sum of match valuation spreads between average and marginal users of the two sides is larger. Although the assumptions of increasing marginal costs and diminishing marginal gains implying under-investment seem reasonable, one should be careful to jump to conclusions. For example, if the investment-cost function is linear and the matching function is convex, $\frac{C_{\alpha}}{M_{\alpha}}$ is a decreasing function and our model predicts over-investment. In addition, for multidimensional user heterogeneities the sum of match valuation spreads in not necessarily positive.

\section{Extension: Endogenous match valuations}

In this section match valuations are no longer considered exogenous. Instead, users are allowed to care about the characteristics of potential trading partners and bargain over the division of the match surplus, which depends on the characteristics of both trading partners.

\subsection{The match value function, user preferences and demand}

Following the matching literature that evaluates the impact of vertically differentiated agents on the matching process (e.g. Becker (1973), Shimer and Smith (2000) and Eeckhout and Kircher $(2010,2011))$, we introduce a match value function $v\left(q^{A}, q^{B}\right)$ that measures the total surplus of a match between an $A$-side user of quality type $q^{A}$ and a $B$-side user of quality type $q^{B}$. Quality types on side $I=A, B$ are distributed over a twice continuously differentiable cdf $F^{I}$ and pdf $f^{I}$ with nonnegative support $[0,+\infty)$. Individual types are private information and distributions common knowledge. We assume that $v$ is continuously differentiable and that for any given trading partner users of both sides can be ranked in their contribution to the match surplus, i.e. $v_{q^{I}} \equiv \partial v / \partial q^{I}>0$. All agents, including the platform, agree over this ranking.

Assume an efficient bargaining process in which users on side $I$ extract a fraction $\beta^{I}$ of total matching surplus. The measure of bargaining power $\beta^{I}$ is assumed exogenous and the same for all users on side $I$. Note that $\beta^{A}+\beta^{B}=1$. Consistent with our assumption of random matching, individual expected utility of users on side $I=A, B$ of patronizing the platform is given by

$$
u^{I}=\beta^{I} v\left(q^{I}, \overline{q^{J}}\right) \frac{M}{N^{I}}-P^{I}
$$

Users on side $I$ participate if $u^{I} \geq 0$ for a given participation fee, matching technology, cross-side 
participation rate and a given expectation of cross-side quality. Expected quality of users on side $I=A, B$ is defined as

$$
\overline{q^{I}} \equiv E\left[q^{I} \mid U^{I} \geq 0\right]=\frac{1}{N^{I}} \int_{\widetilde{q}^{I}}^{+\infty} q^{I} f^{I}\left(q^{I}\right) d q^{I},
$$

where $\widetilde{q^{I}}$ denotes the quality of the marginal user on side $I$. The assumption that $v_{q^{I}}>0 \forall q^{I}$ implies that $v$ is invertible and hence from (13) it follows that $u^{I}=0 \Leftrightarrow q^{I}=v^{-1}\left(\frac{P^{I} N^{I}}{\beta^{I} M}, \overline{q^{J}}\right) \equiv \widetilde{q^{I}}$. Quasi-demand is given by $N^{I}=1-F^{I}\left(\widetilde{q^{I}}\right)$ and inverse demand follows:

$$
P^{I}\left(N^{I}, N^{J}\right)=\beta^{I} v\left(F^{I^{-1}}\left(1-N^{I}\right), \overline{q^{J}}\right) \frac{M}{N^{I}}
$$

From (14) it is clear that any allocation $\left\{N^{A}, N^{B}\right\}$ uniquely pins down prices, so the platform can charge insulating tariffs. The assumption of unidimensional user heterogeneity is crucial for this. In the model, any allocation uniquely pins down marginal and average quality on both sides. This, however, is no longer true for richer specifications of user heterogeneities because a single allocation might result in multiple expectations concerning qualities depending on the "sorting" of marginal users (see Veiga and Weyl (2011)). The impact of multidimensional user heterogeneity on the model outcomes is discussed in the appendix.

\subsection{Private platform pricing}

Proposition 4 summarizes the results obtained from the first-order conditions of the profit maximization problem, given by expression (5).

Proposition 4 In the case of transferable utility, for each side $I=A, B(J \neq I)$, the markup of price over marginal costs of a monopoly private platform is given by

$$
P^{I}-C_{N^{I}}=\mu^{I} \widetilde{v_{q^{I}}^{I}} \frac{M}{N^{I}}-\underbrace{\widetilde{v^{J}} \varphi^{I} \frac{M}{N^{I}}}_{\begin{array}{c}
\text { Subsidize positive cross-side } \\
\text { network externalities }
\end{array}}+\underbrace{\widetilde{v^{I}\left(1-\varphi^{I}\right) \frac{M}{N^{I}}}}_{\begin{array}{c}
\text { Tax negative own-side } \\
\text { network externalities }
\end{array}}+\underbrace{\widetilde{v_{q^{I}}^{J}}\left(\overline{q^{I}}-\widetilde{q^{I}}\right) \frac{M}{N^{I}}}_{\begin{array}{c}
\text { Tax negative } \\
\text { quality externalities }
\end{array}},
$$

Proof. From the expression of quasi-demand $N^{I}=1-F^{I}\left(\widetilde{q^{I}}\right)$ it follows that $\widetilde{q^{I}}=F^{I^{-1}}\left(1-N^{I}\right)$ which illustrates that the platform choice of $N^{I}$ uniquely pins down $\widetilde{q^{I}}$ independent of $N^{J}$. So, $\partial \widetilde{q^{J}} / \partial N^{I}=0$ and $\partial \widetilde{q^{I}} / \partial N^{I}=-1 / f^{I}\left(\widetilde{q^{I}}\right)=-\mu^{I} / N^{I}$. By definition $\overline{q^{I}}$ is also independent of $N^{J}$, so $\partial \overline{q^{J}} / \partial N^{I}=0$ and $\partial \overline{q^{I}} / \partial N^{I}=-\frac{1}{N^{I}}\left(\overline{q^{I}}-\widetilde{q^{I}}\right)$. Using this when differentiating inverse demand, given by (14), with respect to participation rates and substituting this in the first-order conditions of (5) yields the result in proposition 4. The match valuation of the marginal user on side $I$ is now defined as

$$
\widetilde{v^{I}} \equiv \beta^{I} v\left(\widetilde{q^{I}}, \overline{q^{J}}\right)
$$


In addition, $\widetilde{v_{q^{I}}^{I}}$ is a measure of the importance of own quality in the expected match value of marginal users on side $I$, defined as

$$
\widetilde{v_{q^{I}}^{I}} \equiv \beta^{I} \frac{\partial v\left(\widetilde{q^{I}, \overline{q^{J}}}\right)}{\partial \widetilde{q^{I}}}
$$

and $\widetilde{v_{q^{I}}^{J}}$ is a measure of the importance of cross-side quality in the expected match value of marginal users on side $J$, defined as

$$
\widetilde{v_{q^{I}}^{J}} \equiv \beta^{J} \frac{\partial v\left(\overline{q^{I}}, \widetilde{q^{J}}\right)}{\partial \overline{q^{I}}} .
$$

The first term on the right-hand side of expression (15) shows that the classic measure of market power, the inverse hazard rate of demand $\mu^{I}$, in this model is not only proportional to the expected number of transactions $M / N^{I}$ as in proposition 1, but also to the contribution of the marginal user to the expected match surplus of that marginal user $\widetilde{v_{q^{I}}^{I}}$, which by assumption is always positive. This is intuitive, if a marginal user has a higher expected match value due to its own contribution to the surplus, the platform can charge a higher price for that marginal user to be indifferent between participating or not, independent of any network effects.

The second and the third term on the right-hand side of expression (15) are equivalent to the second and third term on the right-hand side of expression (6) in proposition 1 . The platform subsidizes positive cross-side and taxes negative own-side network externalities with respect to the expected match valuation of marginal users. The interpretation is the same as above.

The fourth term demonstrates the main novel insights of accounting for user quality differentiation in a context of random matching. It illustrates how the private platform on each side taxes negative quality externalities. This because a marginal user degrades the average quality of the pool of users on that side and this in turn reduces the expected match valuation of users on the other side. The platform takes this into account in its pricing decision on each side to recover the loss in marginal revenue on the other side. Intuitively, the tax on side $I$ is higher if the spread between the quality of the average user and the marginal user $\left(\overline{q^{I}}-\widetilde{q^{I}}\right)$ is larger and if marginal $J$-side users value the quality of their trading partner more, measured by $\widetilde{v_{q^{I}}^{J}}$.

The intuition is that "more" users on a particular side is not necessarily experienced as "better" by the users on the other side. Instead, there is a trade-off between quantity and quality of the pool of users. For example, in a stylized example of the dating market, women might be happy (and willing to pay more) if a dating club decides to charges a higher entrance fee to men such that very unattractive and unsympathetic men that bring little value to any match are excluded and the average quality of the pool of men to choose from is higher. In this sense, proposition 4 gives a potential explanation for the existence of exclusive nightclubs that charge high entrance fees with the goal to attract only small groups of "high-quality" men and woman. This is more 
likely to occur if cross-side valuations of quality are larger. Similarly, in the online housing market (e.g. LuxuryRealestate.com) and labor market (e.g. TheLadders.com) platforms exist that tend to serve a select group of high quality users at a high price. This phenomenon is hard to explain under the standard assumption in the platform pricing literature that more users on the other side are always perceived as better ${ }^{15}$.

\subsection{Pigouvian pricing and price distortions}

Price distortions are again identified by comparing the pricing decisions of a private platform to those of a social planner. A Pigouvian platform maximizes total social value given by expression (7) in which the average match valuation of participating users on side $I=A, B$ is redefined as

$$
\overline{v^{I}} \equiv \frac{\beta^{I}}{N^{I}} \int_{\widetilde{q^{I}}}^{+\infty} v\left(q^{I}, \overline{q^{J}}\right) f^{I}\left(q^{I}\right) d q^{I}
$$

From the first-order conditions of (7) the markup of price over marginal costs for each side $I=A, B(J \neq I)$ can easily be derived,

$$
P_{P i g}^{I}-C_{N^{I}}=-\underbrace{\overline{v^{J}} \varphi^{I} \frac{M}{N^{I}}}_{\begin{array}{c}
\text { Subsidize positive cross-side } \\
\text { network externalities }
\end{array}}+\underbrace{\overline{v^{I}}\left(1-\varphi^{I}\right) \frac{M}{N^{I}}}_{\begin{array}{c}
\text { Tax negative own-side } \\
\text { network externalities }
\end{array}}+\underbrace{\overline{v_{q^{I}}^{J}}\left(\overline{q^{I}}-\widetilde{q^{I}}\right) \frac{M}{N^{I}}}_{\begin{array}{c}
\text { Tax negative } \\
\text { quality externalities }
\end{array}},
$$

where $\overline{v_{q^{I}}^{J}}$ is a measure of the importance of cross-side quality in the expected match valuation for average users on side $J$, defined as

$$
\overline{v_{q^{I}}^{J}} \equiv \frac{\beta^{J}}{N^{J}} \int_{\widetilde{q^{J}}}^{+\infty} \frac{\partial v\left(\overline{q^{I}}, q^{J}\right)}{\partial \overline{q^{I}}} f^{J}\left(q^{J}\right) d q^{J} .
$$

By adding to and subtracting from expression (15) the terms of the right hand side of expression (16) yields the result in proposition 5.

Proposition 5 For each side $I=A, B(J \neq I)$, the optimal price of the private monopoly platform can be written as, $P^{I}=P_{P i g}^{I}+\mu^{I} \widetilde{v_{q^{I}}^{I}} \frac{M}{N^{I}}+$

$$
\overbrace{\begin{array}{c}
\text { Cross-side } \\
\text { network size distortion }
\end{array}}^{\left(\overline{v^{J}}-\widetilde{v^{J}}\right) \varphi^{I} \frac{M}{N^{I}}}-\underbrace{\text { Spence distortion }}_{\begin{array}{c}
\text { Own-side } \\
\text { network size distortion }
\end{array}} \overline{\left.v^{I}-\widetilde{v^{I}}\right)\left(1-\varphi^{I}\right) \frac{M}{N^{I}}}-\underbrace{\left(\overline{v_{q^{I}}^{J}}-\widetilde{v_{q^{I}}^{J}}\right)\left(\overline{q^{I}}-\widetilde{q^{I}}\right) \frac{M}{N^{I}}}_{\begin{array}{c}
\text { Quality } \\
\text { distortion }
\end{array}} .
$$

\footnotetext{
${ }^{15}$ Our argument for the existence of exclusive platforms is similar to the one developed in Hagiu (2011). A lower cutoff implies lower average quality. For a further discussion on the existence of exclusive market places see also De Fraja and Sákovics (2010) and Halaburda and Piskorski (2011).
} 
Proposition 5 shows that the Spence distortion, on top of the own-side and cross-side network size distortions (which have the same interpretation as in proposition 2), has an additional component, referred to as the quality distortion. The distortion arises because the negative externality, induced by attracting an additional user that degrades the average quality of the pool on that side, is accounted for with respect to the cross-side quality valuation of the marginal user by the private platform and of the average user by the Pigouvian platform.

The direction of the quality distortion crucially depends on the strategic dependence of the quality types in the match value function. Quality types are strategically independent if the crosspartial $v_{q^{A} q^{B}} \equiv \frac{\partial^{2} v}{\partial q^{A} \partial q^{B}}=0$. They are complements if the match value function is a supermodular function, i.e. $v_{q^{A} q^{B}}>0$ and substitutes if it is a submodular function, i.e. $v_{q^{A} q^{B}}<0$. For the ease of interpretation assume that $v_{q^{A} q^{B}}$ is either always positive, negative or zero $\forall q^{A}, q^{B}$. Note that if quality types are strategically independent $\overline{v_{q^{I}}^{J}}=\widetilde{v_{q^{I}}^{J}}$ and the quality distortion disappears. This is for example the case if user utility is assumed to be nontransferable in which case the match valuation only depends on the quality type of the trading partner ${ }^{16}$. If quality types are complements $\overline{v_{q^{I}}^{J}}>\widetilde{v_{q^{I}}^{J}}$ and the distortion is downward and if they are substitutes the distortion is upward.

The intuition behind complementary/substitutable quality types in the match value function is illustrated by the following labor market example. On the one hand, an engineer is expected to be much more productive in a job that requires specific technical and mathematical skills, such as designing a bridge, than when for example a secretarial worker is assigned to this job. Furthermore, when the engineer is assigned to administrative secretarial work, valuable technical skills remain unused. If this is true, the match value function exhibits complementarities and positive assortative matching is optimal, i.e. assign the engineer to design the bridge and let the secretary do the administrative work. The total match value created in the market is then higher than when they are negatively assortatively matched. On the other hand, a typical example (e.g. Eeckhout and Kircher (2011)) of substitutes is the work of a consultant. The gain of a better consultant might be higher if matched to a less productive firm because this firm is likely to be in greater need of a good revision of the organization than a highly productive firm. In this case, negative assortative matching maximizes the total match value in the market.

Note that sorting users of the two sides in the optimal way as described in these examples requires full information about user quality types and the absence of coordination failures. However, in our model the platform is assumed to be a random matchmaker unable to acquire truthful information about quality types before users enter the platform. In terms of sorting efficiency this implies that attracting user groups of higher (lower) average quality on both sides is more efficient if $q^{A}$ and $q^{B}$ are complements (substitutes).

Translated to our results on market distortions this implies that the downward quality distor-

\footnotetext{
${ }^{16}$ This assumption is for example made in Bloch and Ryder (2000), Cañón (2011) and Gomes and Pavan (2011).
} 
tion in the presence of complementary (substitutable) quality types comes from the fact that the tax of the negative quality externalities of the private platform is too low (high) and it would be desirable to attract smaller (larger) groups of higher (lower) average quality on both sides of the market in terms of sorting efficiencies. Intuitively, on each side $I$ the distortion is greater if the spread in quality between the average and marginal user $\overline{q^{I}}-\widetilde{q^{I}}$ is larger (for both complements

and substitutes) and if quality types are greater complements (substitutes), which implies $\overline{v_{q^{I}}^{J}}-\widetilde{v_{q^{I}}^{J}}$ is larger (more negative).

Whether and to which degree quality types are complements or substitutes crucially depends on the specific characteristics of the market in which the platform operates. On a platform like Monster.com that operates in a market for "normal" jobs, one might expect worker and firm types to be complements. However, this is less obvious for a platform like Freelance.com that operates in a specialized market where it might be more efficient to assign better freelancers (e.g. consultants, IT specialists) to less productive firms.

Linking the quality distortions to the sign and magnitude of the cross-partial $v_{q^{A} q^{B}}$ is particularly interesting for the potential empirical implementation of our model. More specifically, in the empirical matching literature recently significant progress has been made (e.g. Eeckhout and Kircher (2011), Lopes de Melo (2011) and Bartolucci and Devicienti (2011)) to estimate the sign and magnitude of the cross-partial in labor markets based on matched firm-worker wage data. These techniques could be used to measure harms of market power of platforms in the labor market, but also for example in the housing market based on housing prices.

To sum up, proposition 5 demonstrates that, for a given unidimensional degree of user heterogeneity on each side, on top of the result of the previous section that trading frictions mitigate price distortions, the classic harms of market power are further reduced if the degree of complementarities between user quality types is larger. Matters become more complicated, however, when allowing for multidimensional user heterogeneities because then both the match valuation spreads $\overline{v^{I}}-\widetilde{v^{I}}$ can also be negative and the composition of the marginal user group on each side comes to play a role (see Veiga and Weyl (2011)), as described in the appendix.

\section{Discussion}

This section discusses some critical assumptions of our approach and links our findings to closely related work.

\subsection{Nonrandom matching and price discrimination}

The recent contribution of Gomes and Pavan (2011) conveniently illustrates the boundaries of our critical assumption of random matching and suggests a complementary approach when the platform can engage in nonrandom matching. They investigate a platform that can implement an incentive compatible mechanism that incentivises participating users to truthfully reveal their qual- 
itative characteristics when they enter the platform. In this case, the platform can nonrandomly match different user groups by their characteristics. In this environment the authors investigate the platform incentives to second-degree price discriminate within user groups ${ }^{17}$. In contrast, we make the opposite assumption that there is no implementable incentive compatible mechanism and that the platform ex ante can do no better than offering a random matching process. Price discrimination based on qualitative user characteristics is irrelevant in our context and we assume the platform can only charge a uniform participation fee within participating groups ${ }^{18}$. Cañón (2011) provides an interesting contribution in between with random meeting and imperfect nonrandom matching through imperfect signaling.

\subsection{Dynamics}

In the present paper, the matching process that occurs at the platform once the two types of user groups have entered the platform is left unspecified. The assumption is made that ex ante users do not know the specifics of the matching process and expect to be randomly matched with any user(s) on the other side. In reality, particularly in one-to-one matching markets, users engage in a search process once they have patronized the platform. In a dynamic setting, some users might reject a match that yields a positive surplus because they expect an offer with a greater surplus in the future if they keep searching. This mechanism might result in ex post sorting of users by type. It is well-known from the matching literature that, depending on the specification of the search process, greater complementarity (substitutability) of quality types in the match value function is more likely to result in efficient sorting outcomes in decentralized search markets. For example, Shimer and Smith (2000) show that even a random search process can result in positive assortative matching if complementarities in the match value function are sufficiently large. In contrast, the present paper shows that the degree of complementarities of user types can also imply a social cost through market distortions if the participation decision of users hinges on the pricing and investment decisions of a private platform. This trade-off is worth further investigation in a dynamic matching model.

\subsection{Platform competition}

A natural question that arises is how the results on market distortions induced by monopoly power are affected when allowing for (imperfect) platform competition. We conjecture that if

\footnotetext{
${ }^{17}$ Gomes and Pavan (2011) focus on a context of many-to-many matching or nonexclusive trade. For a similar analysis of nonrandom matching in a one-to-one matching or exclusive trade setting see, for example, Bloch and Ryder (2000) and De Fraja and Sákovics (2010). In the latter setting network externalities become irrelevant in the platform pricing decisions because in equilibrium each user is matched with a single partner (or not) without uncertainty.

${ }^{18}$ The assumption that the platform can only set up a single market place is crucial for this. When the platform can set up multiple market places price discrimination can also be relevant in a context of random matching. See Damiano and Li (2007) for a detailed analysis.
} 
each user only patronizes a single platform on both sides of the market, the effect of platform competition on the market distortions derived in proposition 2 crucially depends on the returns to scale of the matching function, measured by the sum of matching elasticities on the two sides. More specifically, if $\varphi^{A}+\varphi^{B}<,=,>1$, the matching function exhibits decreasing, constant, increasing returns to scale, respectively. For horizontal platform competition and an increasing returns to scale matching function their might be a trade-off between the classic gains of increased competition and a loss in user surplus because of reduced participation on each platform.

In the presence of user quality differentiation as in the previous section, vertical platform competition can be introduced by characterizing platforms by average user quality on both sides. In a context of random matching, Damiano and Li (2008) study duopoly competition for a special case of our model, absent of network size effects and for a match value function of the specific form $q^{A} q^{B}$. They find that the duopolistic outcome can be less efficient in terms of sorting than the monopoly outcome in spite of servicing more participants. In our more general setting, we expect this result to be amplified in the presence of greater complementarities in user quality types and to be reversed if they are substitutes.

A generalization of the presented model to allow for platform competition (or competition with a decentralized market) in the context of imperfect random matching requires further research attention. Weyl and White (2011) show that the equilibrium concept of insulation extends to arbitrary platform competition in the presence of network externalities under rich specifications of user preferences, however, absent user quality differentiation.

\section{Conclusion}

The present paper compares the optimal pricing (and investment) decisions of a profit and social value maximizing monopoly platform that connects two types of user groups under an imperfect random matching technology. In this environment we establish a direct link between the relative presence of own-side and cross-side network externalities and the amount of trading frictions inherent to the matching process. Consistent with the findings of Weyl (2010) we find that in its optimal pricing decision on each side, the private platform accounts for the private value of network externalities, while the Pigouvian platform accounts for the social value, resulting in a Spence distortion. Particular to our setting is that the Spence distortion has an upward and a downward component and that the weight to each component directly depends on the amount of frictions inherent to the matching process. We show that (for a given degree of unidimensional user heterogeneity in match valuations) on each side the classic harms of monopoly market power are mitigated in the presence of more trading frictions compared to the frictionless benchmark. In terms of investment incentives to reduce the amount of trading frictions we find that for a convex investment-cost function and diminishing marginal gains in matching efficiency, the private platform under-invests compared to the Pigouvian platform. The under-investment is greater if 
the sum of match valuation spreads between the marginal and average user on the two sides is larger.

In the second part of the paper the model is extended to user quality differentiation and users bargain over the division of the match surplus, depending on the quality type of both parties. Greater participation on a particular side now implies a reduction of the average quality on that side, which is negatively valued by cross-side users, implying an additional negative quality externality on top of the classic network size externalities. This results in an additional component in the Spence distortion, referred to as the quality distortion. We show that the quality distortion has the same sign as the cross-partial of the match value function, a measure well-known in the matching literature to quantify the importance of sorting users by type. Greater complements (substitutes) in quality types implies a more downward (upward) quality distortion. For strategically independent quality types the quality distortion disappears.

By introducing a general matching function and match value function in a model of platform pricing, the present paper sets a benchmark to combine theoretical knowledge from both the platform pricing and matching literature. In addition, our approach suggests that methods and techniques of the rich empirical matching literature can be used to identify and estimate important determinants of platform prices and investment decision, like for example the degree of frictions present in the market or the sign and magnitude of the strategic dependence of quality types in the aggregate match surplus.

\section{Appendix: Multidimensional user heterogeneity}

In this appendix we allow for richer specifications of user heterogeneities in valuations of and contributions to the characteristics of the platform service, as in Weyl (2010) and Veiga and Weyl (2011), and discuss the robustness of the results obtained in propositions 1 to 5 . For a tractable presentation we generalize the model step by step. First, we allow for a richer specification of user valuations and revise the results in propositions 1-3. Next, we allow for heterogeneous contributions to our measure of individual quality, temporarily abstracting from network size externalities, in the light of propositions 4-5. Finally, we discuss the difficulties to evaluate a model with both network size and quality externalities in the presence of multidimensional valuations and contributions. More generally, we discuss the limits to the applicability of the solution in Veiga and Weyl (2011) when users are allowed to contribute to multiple characteristics within each user group, even when sufficient instruments are available to the platform.

Multidimensional heterogeneity in user valuations. Assume two types of risk-neutral user groups $I=A, B$ with mass normalized to unity. Users on side $I$ have $\tau^{I}$-dimensional types $\boldsymbol{\theta}^{I} \in \mathbb{R}^{\tau^{I}}$ distributed over the population by a continuous pdf $f^{I}\left(\boldsymbol{\theta}^{I}\right)$. As above, types are private information and distributions common knowledge. We maintain the assumptions of quasi-linear utility and random matching. Expected utility when users decide on participation is specified as 
follows,

$$
u^{I}=v^{I}\left(\frac{M}{N^{I}} ; \boldsymbol{\theta}^{I}\right)-P^{I}
$$

Utility of nonparticipants is normalized to zero. The set of users that patronize the platform on side $I$ is defined as $\Theta^{I} \equiv\left\{\boldsymbol{\theta}^{I}: u^{I} \geq 0\right\}$ and the set of marginal users as $\partial \Theta^{I} \equiv\left\{\boldsymbol{\theta}^{I}: u^{I}=0\right\}$. The fraction of users that participate on side $I$ is denoted by $N^{I}=\int_{\boldsymbol{\theta}^{I} \in \Theta^{I}} f^{I}\left(\boldsymbol{\theta}^{I}\right) d \boldsymbol{\theta}^{I}$ and the density of marginal users by $\widetilde{N^{I}}=\int_{\boldsymbol{\theta}^{I} \in \partial \Theta^{I}} f^{I}\left(\boldsymbol{\theta}^{I}\right) d \boldsymbol{\theta}^{I}$. In what follows we use the simplified notation for integrals $\int_{x \in X} y(x) d x=\int_{X} y$. The matching function $M=M\left(\alpha, N^{A}, N^{B}\right)$ is defined as in section 3.4 , i.e. the platform can influence the efficiency of the matching process through $\alpha$.

Using the terminology of Veiga and Weyl (2011), in this model the platform has three instruments $\boldsymbol{\rho}=\left\{P^{A}, P^{B}, \alpha\right\}$ and two characteristics $\mathbf{K}=\left(N^{A}, N^{B}\right)$. The concept of insulation applies because there are sufficient instruments relative to characteristics. The profit maximization problem of a private platform can be summarized as

$$
\max _{\boldsymbol{\rho}} \pi=P^{A} N^{A}+P^{B} N^{B}-C\left(\alpha, N^{A}, N^{B}\right) \text { subject to } \int_{\Theta^{I}} f^{I}=N^{I}
$$

and the welfare maximization problem of a Pigouvian platform as

$$
\max _{\boldsymbol{\rho}} W=\int_{\Theta^{A}} u^{A} f^{A}+\int_{\Theta^{B}} u^{B} f^{B}+\pi \text { subject to } \int_{\Theta^{I}} f^{I}=N^{I} .
$$

Solving as in Veiga and Weyl (2011), it follows that the optimal private platform choice of participation fee $P^{I}$ for $I=A, B$ and investment $\alpha$ satisfy, respectively,

$$
\begin{aligned}
P^{I}-C_{N^{I}} & =\mu^{I}-\widetilde{v^{J}} \varphi^{I} \frac{M}{N^{I}}+\widetilde{v^{I}}\left(1-\varphi^{I}\right) \frac{M}{N^{I}}, \\
\frac{C_{\alpha^{\pi}}}{M_{\alpha^{\pi}}} & =\widetilde{v^{A}}+\widetilde{v^{B}}
\end{aligned}
$$

where $\widetilde{v^{I}}$ is redefined as the average valuation of marginal users on side I of an additional potential transaction, $\widetilde{v^{I}} \equiv \frac{1}{\widetilde{N}^{I}} \int_{\partial \Theta^{I}} v_{1}^{I} f^{I}$ with $v_{1}^{I}=\partial v^{I} / \partial\left(M / N^{I}\right)$.

Similarly, the optimal welfare maximizing platform choices satisfy

$$
\begin{aligned}
P^{I}-C_{N^{I}} & =-\overline{v^{J}} \varphi^{I} \frac{M}{N^{I}}+\overline{v^{I}}\left(1-\varphi^{I}\right) \frac{M}{N^{I}}, \\
\frac{C_{\alpha^{\pi}}}{M_{\alpha^{\pi}}} & =\overline{v^{A}}+\overline{v^{B}},
\end{aligned}
$$

where $\overline{v^{I}}$ is redefined as the average valuation of all participating users on side $I$ of an additional potential transaction, $\overline{v^{I}} \equiv \frac{1}{N^{I}} \int_{\Theta^{I}} v_{1}^{I} f^{I}$.

These results show that propositions 1-3 are essentially robust for multidimensional heterogeneity in user valuations. The only technical difference is that the inverse hazard rate of demand, 
redefined as $\mu^{I} \equiv N^{I} / \widetilde{N^{I}}$, is no longer proportional to the expected number of transactions. Crucial for the interpretation, however, is that now the average match valuation spread $\overline{v^{I}}-\widetilde{v^{I}}$ on each side can also be negative, which was not the case for unidimensional heterogeneity.

Multidimensional heterogeneity in user valuations and contributions: 2 characteristics, 2 instruments. To illustrate the intuition of the impact of multidimensional heterogeneity on the results in propositions 4 and 5 we temporarily abstract from network size externalities and focus on the quality externalities. To do so, we assume that the expected number of transactions is equal to one, independent of participation rates. We preserve the assumption that users can be ranked in quality in a unidimensional dimension, i.e. $v_{q^{I}}>0$ for both $I=A, B$. However, we do allow users to contribute to this measure of quality in multiple dimensions. The assumption of quasi-linear transferable utility with efficient bargaining is also maintained and expected utility is specified as

$$
u^{I}=\beta^{I} v\left(q^{I}\left(\boldsymbol{\theta}^{I}\right), \overline{q^{J}}\right)-P^{I}
$$

where $q^{I}\left(\boldsymbol{\theta}^{I}\right): \mathbb{R}^{\tau^{I}} \rightarrow \mathbb{R}$, assumed continuous and bounded, denotes the individual quality of a user on side $I$ with type $\boldsymbol{\theta}^{I}$. Expected quality on side $I$ is given by

$$
\overline{q^{I}}=\frac{\int_{\Theta^{I}} q^{I} f^{I}}{\int_{\Theta^{I}} f^{I}}
$$

In this model the platform has two instruments $\boldsymbol{\rho}=\left\{P^{A}, P^{B}\right\}$ and two characteristics $\mathbf{K}=$ $\left(\overline{q^{A}}, \overline{q^{B}}\right)$. To the latter users heterogeneously contribute. Note that the participation rate on each side is not a characteristic users explicitly care about, but instead endogenously follows from the platform choice in instruments and characteristics, $N^{I}\left(P^{I}, \overline{q^{J}}\right)=\int_{\Theta^{I}} f^{I}$ with $\Theta^{I} \equiv\left\{\boldsymbol{\theta}^{I}: u^{I} \geq 0\right\}$.

The profit maximization problem of a private platform can be summarized as

$$
\max _{\boldsymbol{\rho}} \pi=P^{A} N^{A}+P^{B} N^{B}-C\left(N^{A}, N^{B}\right) \text { subject to } \frac{\int_{\Theta^{I}} q^{I} f^{I}}{\int_{\Theta^{I}} f^{I}}=\overline{q^{I}}
$$

and the welfare maximization problem of a Pigouvian platform as

$$
\max _{\boldsymbol{\rho}} W=\int_{\Theta^{A}} u^{A} f^{A}+\int_{\Theta^{B}} u^{B} f^{B}+\pi \text { subject to } \frac{\int_{\Theta^{I}} q^{I} f^{I}}{\int_{\Theta^{I}} f^{I}}=\overline{q^{I}} .
$$

The optimal participation fee on side $I$ for the private platform satisfies

$$
P^{I}-C_{N^{I}}=\mu^{I}-\underbrace{\left(\widetilde{q^{I}}-\overline{q^{I}}\right)}_{\begin{array}{c}
\text { Contribution to quality } \\
\text { of marginal users }
\end{array}} \underbrace{\frac{1}{1-\frac{\widetilde{N^{I}}}{N^{I}} \sigma_{v_{q^{J}}, q^{I}}{\widetilde{N^{J}}}_{N^{J}} \sigma_{v_{q^{I}}^{J}}, q^{J}}}_{\text {Feedback multiplier }} \underbrace{\left(\widetilde{v_{q^{I}}} \frac{N^{J}}{N^{I}}+\widetilde{v_{q^{I}}} \frac{\widetilde{N^{J}}}{N^{J}} \sigma_{v_{q^{I}}, q^{J}}\right)}_{\text {Private value of quality externality }},
$$

where $\sigma_{v_{q^{J}}^{I}, q^{I}}$ denotes the covariance between the valuation of average cross-side quality and the 
contribution to average own-side quality of a typical marginal user on side $I$. Similarly, the optimal participation fee on side $I$ for the Pigouvian platform satisfies

$$
P^{I}-C_{N^{I}}=-\underbrace{\left(\widetilde{q^{I}}-\overline{q^{I}}\right)}_{\begin{array}{c}
\text { Contribution to quality } \\
\text { of marginal users }
\end{array}} \underbrace{\frac{1}{1-\frac{\widetilde{N^{I}}}{N^{I}} \sigma_{v_{q^{J}}, q^{I}} \frac{\widetilde{N^{J}}}{N^{J}} \sigma_{v_{q^{I}}^{J}}, q^{J}}}_{\text {Feedback multiplier }} \underbrace{\left(\overline{v_{q^{I}}^{J}} \frac{N^{J}}{N^{I}}+\overline{v_{q^{J}}^{I}} \frac{\widetilde{N^{J}}}{N^{J}} \sigma_{v_{q^{I}}, q^{J}}\right)}_{\text {Social value of quality externality }} .
$$

Consistent with the findings of Veiga and Weyl (2011), because the individual expected match value depends on own quality (to which users heterogeneously contribute) and expected cross-side quality (which users heterogeneously value) the covariance of these two for marginal users plays a role in the optimal platform pricing decisions. The private platform sets its markup on each side equal to the inverse hazard rate net of the extent that the quality externalities induced by marginal users can be captured by the private platform. As in Veiga and Weyl (2011) the latter can be nicely separated in a contribution component of the marginal users, a feedback effect due to the sorting of marginal users and the private value of the quality externalities. For the Pigouvian platform the inverse hazard rate disappears and the social value of the quality externalities is accounted for.

Note first that, as in proposition 5, if the match value function is strategically independent, all covariances are zero and $\overline{v_{q^{I}}^{J}}=\widetilde{v_{q^{I}}^{J}}$ for both sides, in which case there is no quality distortion. If not, the sign of the distortion depends again on the degree of complementarity or substitutability of quality types, but also on the sign of the covariances. Particular to our setting is that by construction the cross-partial $v_{q^{A} q^{B}}$ and the covariance $\sigma_{v_{q^{J}}^{I}, q^{I}}$ for both sides $I=A, B$ have the same sign. So, if quality types are complements $\left(v_{q^{A} q^{B}}>0\right)$ the quality distortion is unambiguously downward and amplified by the multiplier effects. In other words, in the case of complementary quality types, the result on the quality distortion in proposition 5 is qualitatively robust for multidimensional contributions to our measure of user quality, but the magnitude of the distortion is underestimated due to the multiplier effects. In contrast, in the case of substitutes $\left(v_{q^{A} q^{B}}<0\right)$, the quality distortion is no longer unambiguously upward. Instead, it is more likely to change direction on each side $I=A, B$ the cross-side covariance between the valuation of and contribution to quality of marginal users $\sigma_{v_{q^{I}}^{J}, q^{J}}$ is more negative. Note that, whatever the direction, the distortion is mitigated by the multiplier effects compared to what we find in proposition 5 for unidimensional heterogeneity.

Whether the composition of the group of marginal users attracted by the platform plays a statistical and economic significant role in the market under investigation is essentially an empirical question. Whether one should account for this in the model should be tested for if possible.

Multidimensional heterogeneity in user valuations and contributions: 4 characteristics, 4 instruments. We now generalize the model allowing for both network size and 
quality externalities in the presence of multidimensional heterogeneity in user valuations and contributions. Maintaining the assumptions of quasi-linear utility, random matching, that the match surplus is given by a generic match value function over which matched users efficiently bargain and that users can be unidimensionally ranked in quality, expected utility is specified as

$$
u^{I}=\beta^{I} v\left(q^{I}\left(\boldsymbol{\theta}^{I}\right), \overline{q^{J}}\right) \frac{M}{N^{I}}-P^{I}
$$

where $q^{I}\left(\boldsymbol{\theta}^{I}\right): \mathbb{R}^{\tau^{I}} \rightarrow \mathbb{R}$, assumed continuous and bounded, denotes the individual quality of a user on side $I$ with type $\boldsymbol{\theta}^{I}$. Expected quality $\overline{q^{I}}$ is defined as above.

In this model the platform has three instruments $\boldsymbol{\rho}=\left\{P^{A}, P^{B}, \alpha\right\}$ and four characteristics $\mathbf{K}=$ $\left(N^{A}, N^{B}, \overline{q^{A}}, \overline{q^{B}}\right)$. A first problem immediately arises: the equilibrium concept of insulation is only applicable if the platform has at least as much instruments as characteristics, as shown by Veiga and Weyl (2011). In section 4 this was not an issue because the assumption on unidimensional user heterogeneity implied that the platform allocation choice $\left\{N^{A}, N^{B}\right\}$ simultaneously uniquely pinned down expected qualities $\left\{\overline{q^{A}}, \overline{q^{B}}\right\}$ and insulating prices $\left\{P^{A}, P^{B}\right\}$. This is no longer true in a model with richer heterogeneity because for a given participation rate $N^{I}$ the expected quality depends on the sorting of multiple marginal users and no longer on the cutoff of a single marginal user.

This issue can be resolved under the assumption that the platform has an additional instrument at its disposal. For example, the platform might influence the aggregate number of transactions in a side-specific way, such that $M=M\left(\alpha^{A}, \alpha^{B}, N^{A}, N^{B}\right)$. A specific form one can assume is $M=\left(N^{A}\right)^{\alpha^{A}}\left(N^{B}\right)^{\alpha^{B}}$ in which case the platform can directly choose the matching elasticities for each side. Alternatively, the platform might charge a transaction fee or a commission conditional on matching. The proper assumptions on instruments of course depend on the properties of the market under investigation and should be chosen appropriately.

A more fundamental methodological problem, however, is to come to a tractable solution. Even when sufficient instruments are available to the platform, if users are allowed to contribute to multiple characteristics within different user groups, a case not considered by Veiga and Weyl (2011), the general solution offered by the authors does not apply ${ }^{19}$. A solution exists, however, the contribution to characteristics of marginal users and the value (social or private) of the externalities can no longer be separated as was the case for the above example of 2 characteristics and 2 instruments. The statistical and economic significance of heterogeneity in the group of marginal users should be empirically tested for (if possible) and the trade-off should be made to incorporate this in the model at the cost of less analytical tractability.

\footnotetext{
${ }^{19}$ Technically, this is the case because the matrices with valuations for instruments $\overline{u_{\rho}}$ and $\widetilde{u_{\rho}}$ are not invertible.
} 


\section{References}

Anderson, S. and de Palma, A. 2009. "Information Congestion" RAND Journal of Economics, Vol. 40, No 4, Winter 2009, 688-709.

Armstrong, M. 2006. "Competition in two-sided markets" RAND Journal of Economics, Vol. 37, No 3, Autumn 2006, 668-691.

Bartolucci, C. and Devicienti, F. 2011. "Identifying Sorting in Practice" Working Paper.

Becker, S.G. 1973. "A Theory of Marriage: Part I" Journal of Political Economy, 81(4), 1973, 813-46.

Belleflamme, P. and Toulemonde, E. 2009. "Negative Intra-Group Network Externalities In Two-Sided Markets" International Economic Review, Vol. 50, No. 1, February 2009.

Bloch, F. and Ryder, H. 2000. "Two-sided search, marriages and matchmakers" International Economic Review, Vol. 41, pp. 93-115.

Cañón, C. 2011. "Matching and Information Provision in Two-Sided Markets" Working Paper.

Caillaud, B. and Jullien, B. 2003. "Chicken and egg: competition among intermediation service providers" RAND Journal of Economics, Vol. 34, No 2, Summer 2003, 309-328.

Cheng, K-P. and Huang, Y-C. 2009. "A search-matching model of the two-sided markets" Working Paper.

Damiano, E. and Li, H. 2007. "Price discrimination and efficient matching" Economic Theory, Vol. 30, pp. 243-263.

Damiano, E. and Li, H. 2008. "Competing matchmaking" Journal of the European Economic Association, Vol. 6, pp. 789-818.

De Fraja, G. and Sákovics, J. 2010. "Exclusive nightclubs and lonely hearts columns: non-monotone participation in optional intermediation" Working Paper.

Eeckhout, J. and Kircher, P. 2010. "Sorting and Decentralized Price Competition." Econometrica 78(2), 2010, 539-574.

Eeckhout, J. and Kircher, P. 2011. "Identifying Sorting - In Theory" Review of Economic Studies (2011) 78(3): 872-906.

Evans, D. and Schmalensee, R. 2010. "Failure to Launch: Critical Mass in Platform Businesses" Review of Network Economics, Vol. 9: Iss. 4, Article 1.

Gomes, R. and Pavan, A. 2011. "Price Discrimination in Many-to-Many Matching Markets" Working Paper.

Hagiu, A. 2011. "Quantity vs Quality: Exclusion by Platforms with Network Effects" Working Paper.

Halaburda, H. and Piskorski M.J. 2011. "Platforms and limits to network effects" Working Paper. 
Lopes de Melo, R. 2011. "Sorting in the Labor Market: Theory and Measurement" Working Paper.

Petrongolo, B. and Pissarides C. 2001. "Looking into the black box: a survey of the matching function" Journal of Economic Literature, Vol. XXXIX (June 2001), 390-431.

Pissarides, C. 2000. "Equilibrium Unemployment Theory" Second edition, Cambridge, MA: MIT Press, 2000 (reprinted 2007).

Rochet, J-C. and Tirole, J. 2003. "Platform Competition in Two-Sided Markets" Journal of the European Economic Association, Vol. 1, 990-1029.

Rochet, J-C. and Tirole, J. 2006. "Two-sided markets: a progress report" RAND Journal of Economics, Vol. 37, No 3, Autumn 2006, 645-667.

Rogerson, R., Shimer, R. and Wright, R. 2005. "Search-Theoretic Models of the Labor Market: A Survey" Journal of Economic Literature, Vol. XLIII (December 2005), pp. 959-988.

Shimer, R. and Smith, L. 2000. "Assortative Matching and Search." Econometrica 68, 2000, 343-369.

Spence, M.A. 1975. "Monopoly, Quality, and Regulation" The Bell Journal of Economics, 6, 417-429.

Trégouët, T. 2010. "Competition in matching technologies" Working Paper.

Veiga, A. and Weyl, G. 2011. "Multidimensional Heterogeneity and Platform Design" Working Paper.

Viecens, M. F. 2009. "Pricing Strategies in Two-Sided Platforms: the Role of Sellers' Competition" Working Paper FEDEA 2009-11.

Weyl, G. 2010. "A price theory of multi-sided platforms" American Economic Review 100 (September 2010), 1642-1672.

Weyl, G. and White, A. 2011. "Insulated platform competition" Working paper. 
Copyright (c) 2011 @ the author(s). Discussion papers are in draft form. This discussion paper is distributed for purposes of comment and discussion only. It may not be reproduced without permission of the copyright holder. Copies of working papers are available from the author. 